\title{
Need for equity in treatment of substance use among Indigenous people in Canada
}

\author{
Karen A. Urbanoski PhD
}

— Cite as: CMAJ 2017 November 6;189:E1350-1. doi: 10.1503/cmaj.171002

See related article at www.cmaj.ca/lookup/doi/10.1503/cmaj.160778

I n Canada, as in many other countries, Indigenous people have poorer health, on average, than non-Indigenous people, by most measures of health and well-being. Indigenous people (comprising First Nations, Métis and Inuit people in Canada) also carry a disproportionate burden of the harms related to substance use. The linked study reports new findings from the Cedar Project, an ongoing study of Indigenous youth in British Columbia, ${ }^{1}$ which provide further evidence of elevated mortality rates among Indigenous youth who use drugs. These findings serve as a stark reminder of the need for tailored services and policies that are better able to meet the needs of Indigenous people in Canada. Variability in health and well-being across Indigenous communities serves to highlight potential solutions to reduce these disparities.

The health inequities experienced by Indigenous people represent the embodiment of the structural and systemic disadvantages that result from the enduring legacy of colonization, which have served to displace and disconnect them from their communities, families and culture. ${ }^{2,3}$ The stigma and discrimination surrounding substance use, particularly illicit drugs, serves to further marginalize people who have structural disadvantages, including Indigenous people, and to compound the negative impacts on health related to substance use.

In Canada, much of the existing evidence on health disparities experienced by people who use drugs comes from research conducted in the Downtown Eastside neighbourhood of Vancouver. In this community, rates of HIV and hepatitis C virus infection, and homelessness are higher among Indigenous people who use drugs compared to non-Indigenous people who use drugs., ${ }^{3,4}$ Evidence from prospective studies shows that people who identify as Indigenous are less likely to receive treatment for substance use (including but not limited to opioid agonist treatment) and, if they do access treatment, they are more likely to drop out. ${ }^{5,6}$ Currently in $\mathrm{BC}$, Indigenous communities are being disproportionately affected by the opioid overdose crisis: recent analyses of provincial administrative data show that First Nations people are five times more likely to overdose and three times more likely to die from overdose than non-First Nations people. ${ }^{7}$

\section{KEY POINTS}

- Indigenous people have poorer health, on average, than non-Indigenous populations and carry a disproportionate burden of the harms related to substance use, resulting from structural and systemic disadvantages caused by colonization.

- Potential solutions to reduce health disparities may include individuals being connected to traditional culture.

- Evidence supports the promise of culturally based and community-owned services for promoting individual and community health, and reducing health disparities.

How Indigenous identity is defined and measured in research has implications for study findings and interpretation. Misclassification errors and biases in nonresponse and noncoverage in Canada's main sources of population health data (e.g., the Census, provincial administrative health data and population health surveys) have led to the systematic underestimation of health inequities between Indigenous and non-Indigenous people. ${ }^{8}$ Existing data sources cannot produce unbiased statistics that can convey potentially critical distinctions between groups defined by geography, government policy or self-identity. The result is an evidence base that provides an unclear understanding of the health of Indigenous communities and people, and is not suited to informing population and community health interventions and policies to reduce health disparities.

Population-based estimates do not tell (and are not meant to tell) the full story and need to be supplemented with indicators generated from small-area studies of regions or communities to yield important evidence to inform policy and practice. For example, substantially higher rates of suicide among Indigenous, relative to non-Indigenous, youth at the provincial level in BC obscure wide variation in rates across Indigenous communities in the province. ${ }^{9}$ Examination of regional differences could give insight into the underpinnings of local resiliency, for example, that could inform the development of policies and programs to support health in communities where they are needed. 
Connection to Indigenous culture has been shown to play a role in reducing disparities in health, including substance-related harms. In the study of youth suicide mentioned previously, community suicide rates in $\mathrm{BC}$ varied inversely with measures of the strength of cultural identity and self-governance in Indigenous communities, which suggests a connection between culture and community health. ${ }^{9}$ A systematic review of 19 studies evaluated evidence of the effectiveness of Indigenous culture-based interventions for substance use, typically integrated with Western intervention approaches. ${ }^{10}$ The authors concluded that culturebased interventions were associated with positive improvements in health and well-being, including reduced substance use and related harms, but that the evidence base was lacking because of the use of relatively weak study designs. Recommendations for future studies include providing more detail on interventions and their intended functions, paying greater attention to differences across subgroups defined by sociodemographic characteristics and Indigenous identities, and using stronger study designs, both qualitative and quantitative, with a focus on elucidating the causal mechanisms through which culture influences the recovery process. Research is also needed to determine whether the availability of culture-based interventions results in improved access to services for treatment of substance use and reduces the disparity in drop-out rates between Indigenous and nonIndigenous patients.

More generally, frameworks for addressing health and health care inequities among Indigenous people (generated through document review and participatory research approaches) share an emphasis on cultural safety and trauma-informed practice. ${ }^{11,12}$ Strategies for promoting equity in access to health care emphasize meaningful engagement and partnerships with Indigenous communities, community ownership of services, and the importance of integrated holistic services that attend to the social determinants of health. It is notable that these same strategies have emerged in the literature about systems planning and service delivery for substance use in Canada and elsewhere. Finally, and picking up on the limitations of existing data sources noted previously, issues of Indigenous ownership and participation extend to the development and surveillance of health indicators with which progress is measured. ${ }^{8}$

The further development of culturally based and communityowned services holds promise for achieving gains in population health and reducing disparities among Indigenous communities. However, the need for tailored services does not negate the need for broader structural and systemic change to address socioeconomic disparities and to remove the social and political barriers that continue to have a negative impact on the health of Indigenous communities in Canada.

\section{References}

1. Jongbloed K, Pearce ME, Pooyak S, et al. The Cedar Project: mortality among young Indigenous people who use drugs in British Columbia. CMAJ 2017;189:E1352-9.

2. Hackett C, Feeny D, Tompa E. Canada's residential school system: measuring the intergenerational impact of familial attendance on health and mental health outcomes. J Epidemiol Community Health 2016;70:1096-1105.

3. Adelson E. The embodiment of inequity: health disparities in Aboriginal Canada. Can J Public Health 2005;96(Suppl2):S45-S61.

4. Wood E, Montaner JS, Li K, et al. Burden of HIV infection among aboriginal injection drug users in Vancouver, British Columbia. Am J Public Health 2008;98:515-9.

5. Li X, Sun H, Marsh DC, et al. Factors associated with pretreatment and treatment dropouts: comparisons between Aboriginal and non-Aboriginal clients admitted to medical withdrawal management. Harm Reduct J 2013;10:38.

6. Wood E, Li K, Palepu A, et al. Sociodemographic disparities in access to addiction treatment among a cohort of Vancouver injection drug users. Subst Use Misuse 2005;40:1153-67.

7. Overdose data and First Nations in BC: preliminary findings. Vancouver: First Nations Health Authority; 2017. Available: www2.gov.bc.ca/assets/gov/overdose -awareness/fnha_overdosedataandfirstnationsinbc_preliminaryfindings_finalweb _july20.pdf (accessed 2017 Aug. 23).

8. Smylie J, Firestone M. Back to the basics: Identifying and addressing underlying challenges in achieving high quality and relevant health statistics for indigenous populations in Canada. Stat J IAOS 2015;31:67-87.

9. Chandler MJ, Lalonde C. Cultural continuity as a hedge against suicde in Canada's First Nations. Transcult Psychiatry 1998;35:191-219.

10. Rowan M, Poole N, Shea B, et al. Cultural interventions to treat addictions in Indigenous populations: findings from a scoping study. Subst Abuse Treat Prev Policy 2014;9:34.

11. Browne AJ, Varcoe C, Lavoie J, et al. Enhancing health care equity with Indigenous populations: evidence-based strategies from an ethnographic study. BMC Health Serv Res 2016;16:544.

12. Davy C, Harfield S, McArthur A, et al. Access to primary health care services for Indigenous peoples: a framework synthesis. Int J Equity Health 2016;15:163.
Competing interests: Karen Urbanoski holds a Canadian Institutes of Health Research Canada Research Chair in Substance Use, Addictions, and Health Services.
Affiliations: Centre for Addictions Research of BC; School of Public Health and Social Policy, University of Victoria, Victoria, BC

This article was solicited and has not been peer reviewed.
Correspondence to: Karen Urbanoski,

Correspondence to:
urbanosk@uvic.ca 